\title{
Threatened fishes of the world: Coreius septentrionalis (Nichols, 1925) (Cyprinidae)
}

\author{
Denghang Yu • Shiyuan Gong $•$ Renhai Peng
}

Received: 26 February 2009 / Accepted: 19 October 2009 /Published online: 30 October 2009

(C) Springer Science + Business Media B.V. 2009

Keywords Coreius septentrionalis Nichols .

Threatened fishes

Common name: Pigeon fish (Chinese). Conservation status: Threatened in China Red Data Book (Yue and Chen 1998) and China Species Red List (Wang and Xie 2004). Identification: A freshwater fish; long cylindrical body, small and slightly flattened head, horseshoe-shaped mouth with sharp and prominent lips, a pair of rough long barbels, small round eyes with red iris (Li 1990). Lateral line scales 55-56, D: 3, 7; P: 1, 18; V: 1, 7; A: 3, 6 (Wu et al. 1964). This illustration is from Chinese Cyprinidae Records (Wu et al. 1964). Distribution: Only found in the Yellow River in China. Habitat and ecology: Lives in the middle layer of the river, where the water flow is moderate and with a lot of gravel on the substrate (Peng 2007). Reproduction: Mature adults migrate upstream to breed in the spawning grounds in areas with fast currents in May to July. Threats: Popula-

D. Yu $(\bowtie) \cdot S$. Gong $\cdot$ R. Peng

College of Fisheries, Huazhong Agricultural University, Wuhan 430070, China

e-mail: yudenghang1985@163.com

D. $\mathrm{Yu} \cdot \mathrm{S}$. Gong $\cdot \mathrm{R}$. Peng

School of Biotechnology and Food,

Anyang Institute of Technology,

Anyang 455000, China

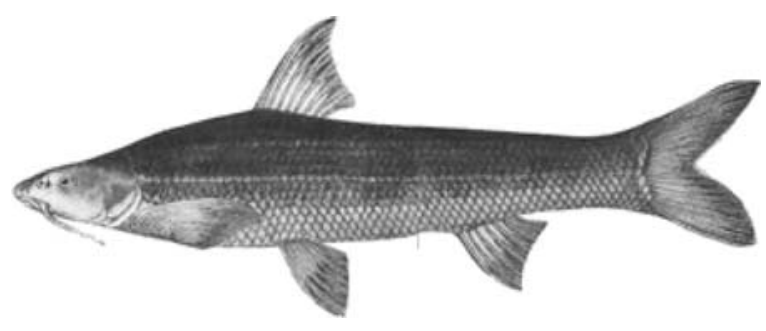

tions have declined seriously due to overfishing and water pollution (Li 1990). Conservation action: No specific action has been adopted for this fish. Conservation recommendation: Water pollution should be controlled; spring fishing prohibition period should be adopted; artificial breeding and hatching should be attempted; and protection of fish resources should be progressively achieved.

\section{References}

Li ZH (1990) Threatned fish: Coreius septentrionalis Nichols (in Chinese). China Nature 2

Peng RH (2007) Culture test of Coreius septentrionalis Nichols (in Chinese). Scientific Fish Farming. Vol. 6

Yue PQ, Chen YY (1998) China red data book of endangered animals: pisces. Science, Beijing (in Chinese with English translation)

Wang S, Xie Y (2004) China species red list. Red list, vol. 1. Higher Education, Beijing in Chinese

Wu XW et al (1964) Chinese cyprinidae records. Science and Technology, Shanghai, p 504 in Chinese 